\title{
Oscillating Free Convection Flow between Two Parallel Plates with Mass Diffusion
}

\author{
Fasihah Zulkiflee ${ }^{1}$, Ahmad Qushairi Mohamad ${ }^{1}$, Mohd Rijal Ilias², Sharidan Shafie ${ }^{1, *}$ \\ ${ }^{1}$ Department of Mathematical Sciences, Faculty of Science, Universiti Teknologi Malaysia, Malaysia \\ ${ }^{2}$ Department of Mathematical Sciences, Faculty of Computer and Mathematical Sciences, Universiti Teknologi MARA, Malaysia
}

Received July 1, 2019; Revised August 22, 2019; Accepted September 22, 2019

Copyright@2019 by authors, all rights reserved. Authors agree that this article remains permanently open access under the terms of the Creative Commons Attribution License 4.0 International License

\begin{abstract}
This paper studied unsteady free convection flow between two parallel plates with mass diffusion. One of the plates is considered oscillating. Appropriate non-dimensional variables are used to reduce the dimensional governing equations along with imposed initial and boundary conditions. The exact solution to velocity, temperature and concentration profiles are obtained using the Laplace Transform technique. The graphical results of the solutions are presented to illustrate the behavior of the fluid flow with the influence of Schmidt number, Prandtl number, oscillating parameter, Grashof and mass Grashof number. The corresponding expressions for skin friction, Nusselt number and Sherwood number are also calculated. It is observed that increasing Prandtl and Schmidt numbers will increased the Nusselt number but decreased the skin friction.
\end{abstract}

Keywords Free Convection, Mass Diffusion, Oscillating, Parallel Plates

\section{Introduction}

The fluid flows between parallel plates have received much attention due to the various applications involving heat transfer. It has various applications such as in petroleum industry, purification of crude oil, pumps accelerators and power generators [1]. Researchers have taken a great interest investigated problem regarding free convection flow between two parallel plates [2-4]. Singh investigated transient natural convection between two vertical walls heated/cooled asymmetrically [5]. Narahari [6] investigated natural convection flow in vertical channel with ramped wall temperature at one boundary while Paul [2] investigated transient free convective flow in vertical channel with constant temperature and constant heat flux. In other research, Jha [7] investigate free convection heat and mass transfer flow in a vertical channel with Dufour effect and then extended their research with diffusion-thermo effects on free convective heat and mass transfer flow in a vertical channel with symmetry boundary condition [8].

Oscillatory flow between parallel plates has received some attention of the researchers as because researchers found that oscillatory plates can higher the rates of heat transfer [9]. There are many researchers considering oscillation in their research such as [10-12] but not many considering oscillations in between two parallel plates. The literature survey shows that researches on oscillating flow between two parallel plates are very few. Reddy [13] investigated unsteady MHD free convection oscillatory Coette flow through a porous medium with periodic wall temperature in presence of chemical reaction and thermal radiation. While MHD oscillatory flow through a porous channel saturated with porous medium was investigated by Falade [14]. Bunyonyo [9] investigated unsteady oscillatory Coette flow between vertical plates with constant radiative heat flux. Their research shows that the oscillatory parameter played a greater role in causing the fluid to oscillate inside the Coette channel.

Motivated by above investigations, the present analysis is to investigate oscillatory free convection flow between two parallel plates with mass diffusion. This problem will be solved using Laplace Transform method to obtain exact solution.

\section{Materials and Methods}

Consider an unsteady free convection flow between two parallel plates with mass diffusion. Oscillating plate at $y^{\prime}=0$ is considered. The configuration flow of the problem is presented in Figure 1. The flow is governed by the following equations under the usual Boussinesq's approximations: 


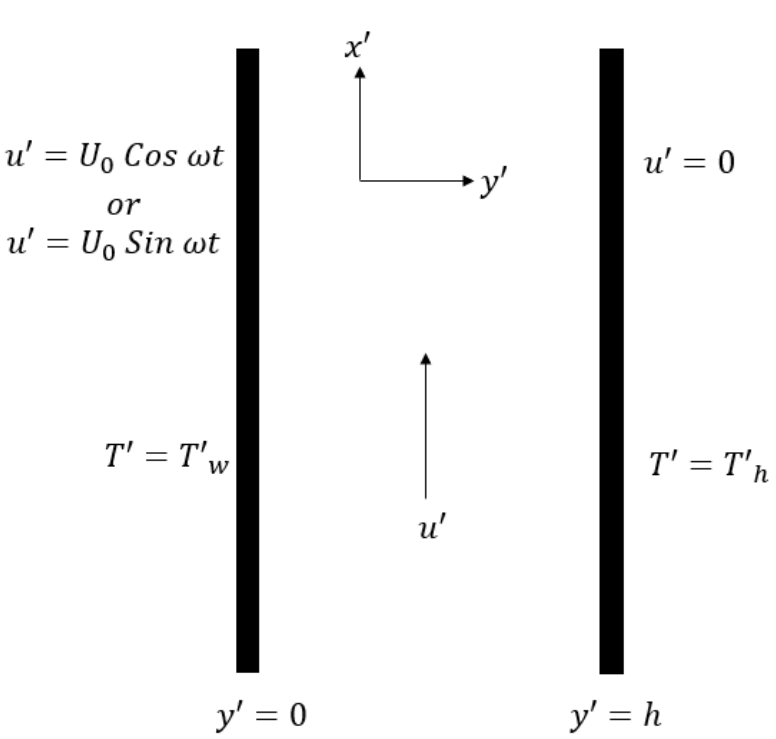

Figure 1. Flow configuration of the problem

$$
\begin{gathered}
\frac{\partial u^{\prime}}{\partial t^{\prime}}=v \frac{\partial^{2} u^{\prime}}{\partial y^{2^{\prime}}}+g \beta\left(T^{\prime}-T_{h}^{\prime}\right)+g \beta\left(C^{\prime}-C_{h}^{\prime}\right) \\
\rho C_{p} \frac{\partial T^{\prime}}{\partial t^{\prime}}=k \frac{\partial^{2} T^{\prime}}{\partial y^{2^{\prime}}} \\
\frac{\partial C^{\prime}}{\partial t^{\prime}}=D \frac{\partial^{2} C^{\prime}}{\partial y^{2^{\prime}}}
\end{gathered}
$$

Subject to

$$
\begin{aligned}
& t^{\prime} \leq 0: u^{\prime}=0, T^{\prime}=T_{h}^{\prime}, C^{\prime}=C_{h}^{\prime}, 0 \leq y^{\prime} \leq h \\
& t^{\prime} \geq 0: \\
& u^{\prime}=U_{0} \cos \omega \mathrm{t} \text { and } U_{0} \sin \omega \mathrm{t}, T^{\prime}=T_{w}^{\prime}, C^{\prime}=C_{w}^{\prime}, y^{\prime}=0 \\
& u^{\prime}=0, T^{\prime}=T_{h}^{\prime}, C^{\prime}=C_{h}^{\prime}, y^{\prime}=0
\end{aligned}
$$

Here $u$ ' is the velocity of the fluid, $g$ is the acceleration due to gravity, $\beta$ is the volumetric coefficient of thermal expansion, $t$ ' is the time, $\mathrm{h}$ is the distance between two vertical plates, $T$ ' is the temperature of the fluid, $T{ }^{\prime}{ }_{h}$ is the temperature of the plate at $y^{\prime}=h, \beta^{*}$ is the volumetric coefficient of concentration expansion, $C^{\prime}$ is the species concentration of the fluid, $C^{\prime}{ }_{h}$ is the species concentration at the plate $y^{\prime}=h, v$ is the kinematic viscosity, $y^{\prime}$ is the coordinate axis normal to the plates, $\rho$ is the density, $C_{p}$ is the specific heat at constant pressure, $k$ is the thermal conductivity of the fluid, $D$ is the mass diffusion coefficient, $T^{\prime}{ }_{w}$ is the temperature of the plate at $y^{\prime}=0$ and $C^{\prime}{ }_{w}$ is the species concentration of the plate $y^{\prime}=0$.The non-dimensional quantities are introduced:

$$
\begin{aligned}
& y=\frac{y^{\prime}}{h}, \quad t=\frac{t^{\prime} v}{h^{2}}, \quad u=\frac{u^{\prime}}{U_{0}}, \\
& T=\frac{T^{\prime}-T_{h}^{\prime}}{T_{w}^{\prime}-T_{h}^{\prime}}, \quad C=\frac{C^{\prime}-C_{h}^{\prime}}{C_{w}^{\prime}-C_{h}^{\prime}}, \quad \mu=\rho v
\end{aligned}
$$

Then, from equation (5), equations (1)-(3) and boundary conditions (4) become:

$$
\frac{\partial u}{\partial t}=\frac{\partial^{2} u}{\partial y^{2}}+G r \theta+G m C
$$

$$
S c \frac{\partial C}{\partial t}=\frac{\partial^{2} C}{\partial y^{2}}
$$

Subject to

$$
\begin{aligned}
& t \leq 0: u=0, \quad \theta=0, \quad C=0, \quad 0 \leq y \leq 1 \\
& t \geq 0: \\
& u=\cos \omega t \text { and } \sin \omega t, \quad \theta=1, C=1, y=0 \\
& u=0, \quad \theta=0, \quad C=0, \quad y=1
\end{aligned}
$$

where,

$$
\begin{gathered}
G r=\frac{g \beta h^{2}}{v U_{0}}\left(T_{w}^{\prime}-T_{h}^{\prime}\right), \quad G m=\frac{g \beta^{*} h^{2}}{v U_{0}}\left(C_{w}^{\prime}-C_{h}^{\prime}\right), \\
S c=\frac{v}{D}, \quad \operatorname{Pr}=\frac{\mu C_{p}}{k},
\end{gathered}
$$

are the thermal Grashof number, mass Grashof number, Schmidt number and Prandtl number respectively. Here $\omega t$ is the oscillating parameter.

\section{Results and Discussion}

The governing equations (6)-(8) with boundary conditions (9) are solved in exact form by using Laplace transform technique and solutions for velocity with sine and cosine cases, temperature and concentration in the transform $(y, q)$ plane are given by 


$$
\begin{aligned}
& \bar{u}_{c}=\sum_{n=0}^{\infty}\left\{\begin{array}{l}
\frac{a_{3}}{q^{2}} e^{-a \sqrt{q}}-\frac{a_{3}}{q^{2}} e^{-b \sqrt{q}}+\frac{1}{2(q-i \omega)} e^{-a \sqrt{q}} \\
-\frac{1}{2(q-i \omega)} e^{-b \sqrt{q}}+\frac{1}{2(q+i \omega)} e^{-a \sqrt{q}} \\
-\frac{1}{2(q+i \omega)} e^{-b \sqrt{q}}-\frac{a_{1}}{q^{2}} e^{-a \sqrt{\operatorname{Pr} q}}+\frac{a_{1}}{q^{2}} e^{-b \sqrt{\operatorname{Pr} q}} \\
-\frac{a_{2}}{q^{2}} e^{-a \sqrt{S c q}}+\frac{a_{2}}{q^{2}} e^{-b \sqrt{S c q}}
\end{array}\right\} \\
& \bar{u}_{s}=\sum_{n=0}^{\infty}\left\{\begin{array}{l}
\frac{a_{3}}{q^{2}} e^{-a \sqrt{q}}-\frac{a_{3}}{q^{2}} e^{-b \sqrt{q}}+\frac{1}{2(q-i \omega)} e^{-a \sqrt{q}}-\frac{1}{2(q-i \omega)} e^{-b \sqrt{q}} \\
-\frac{1}{2(q+i \omega)} e^{-a \sqrt{q}}+\frac{1}{2(q+i \omega)} e^{-b \sqrt{q}}-\frac{a_{1}}{q^{2}} e^{-a \sqrt{\mathrm{Pr} q}} \\
+\frac{a_{1}}{q^{2}} e^{-b \sqrt{\mathrm{Pr} q}}-\frac{a_{2}}{q^{2}} e^{-a \sqrt{s c q}}+\frac{a_{2}}{q^{2}} e^{-b \sqrt{s c q}}
\end{array}\right\} \\
& \bar{\theta}=\sum_{n=0}^{\infty}\left\{\frac{1}{q} e^{-a \sqrt{\operatorname{Pr} q}}-\frac{1}{q} e^{-b \sqrt{\operatorname{Pr} q}}\right\} \\
& \bar{C}=\sum_{n=0}^{\infty}\left\{\frac{1}{q} e^{-a \sqrt{S c q}}-\frac{1}{q} e^{-b \sqrt{S c q}}\right\}
\end{aligned}
$$

Then, the Laplace inversion in $(y, t)$ obtained is as follows,

$$
\begin{aligned}
& u_{c}=\sum_{n=0}^{\infty}\left\{\begin{array}{l}
a_{3}\left(f_{1}(a, t)-f_{1}(b, t)\right)+\frac{e^{i \omega t}}{4}\left(f_{2}(a, t)-f_{2}(b, t)\right) \\
+\frac{e^{i \omega t}}{4}\left(f_{3}(a, t)-f_{3}(b, t)\right)+a_{1}\left(-f_{4}(a \sqrt{\operatorname{Pr}}, t)\right. \\
\left.+f_{4}(b \sqrt{\operatorname{Pr}}, t)\right)+a_{1}\left(-f_{4}(a \sqrt{S c}, t)+f_{4}(b \sqrt{S c}, t)\right)
\end{array}\right\} \\
& u_{s}=\sum_{n=0}^{\infty}\left\{\begin{array}{l}
a_{3}\left(f_{1}(a, t)-f_{1}(b, t)\right)+\frac{e^{i \omega t}}{2(2 i)}\left(f_{2}(a, t)-f_{2}(b, t)\right) \\
+\frac{e^{i \omega t}}{2(2 i)}\left(-f_{3}(a, t)+f_{3}(b, t)\right)+a_{1}\left(-f_{4}(a \sqrt{\operatorname{Pr}}, t)\right. \\
\left.+f_{4}(b \sqrt{\operatorname{Pr}}, t)\right)+a_{1}\left(-f_{4}(a \sqrt{S c}, t)+f_{4}(b \sqrt{S c}, t)\right)
\end{array}\right\} \\
& \theta(y, t)=\sum_{n=0}^{\infty} f_{4}(a \sqrt{\operatorname{Pr}}, t)-f_{4}(b \sqrt{\operatorname{Pr}}, t) \\
& C(y, t)=\sum_{n=0}^{\infty} f_{4}(a \sqrt{S c}, t)-f_{4}(b \sqrt{S c}, t)
\end{aligned}
$$

where,

$$
a=2 n+y, b=2 n+2-y,
$$

$$
a_{1}=\frac{G r}{\operatorname{Pr}-1}, \quad a_{2}=\frac{G m}{S c-1}, \quad a_{3}=\frac{G r}{\operatorname{Pr}-1}+\frac{G m}{S c-1},
$$

$$
\begin{gathered}
f_{1}=\left(\frac{z^{2}}{2}+t\right) \operatorname{Erfc}\left(\frac{z}{2 \sqrt{t}}\right)-z \sqrt{\frac{t}{\pi}} e^{-\frac{z^{2}}{4 t}} \\
f_{2}=e^{z \sqrt{i \omega}} \operatorname{Erfc}\left(\frac{z}{2 \sqrt{t}}+\sqrt{i \omega t}\right)+e^{-z \sqrt{i \omega}} \operatorname{Erfc}\left(\frac{z}{2 \sqrt{t}}-\sqrt{i \omega t}\right) \\
f_{3}=e^{z \sqrt{-i \omega}} \operatorname{Erfc}\left(\frac{z}{2 \sqrt{t}}+\sqrt{-i \omega t}\right)+e^{-z \sqrt{-i \omega}} \operatorname{Erfc}\left(\frac{z}{2 \sqrt{t}}-\sqrt{-i \omega t}\right) \\
f_{4}=\operatorname{Erfc}\left(\frac{z}{2 \sqrt{t}}\right) .
\end{gathered}
$$

Here the subscripts $c$ and $s$ in above equations refer to cosine and sine oscillations of the plate. From the solutions above, Skin friction, Nusselt number and Sherwood number are calculated by using the following expression,

Skin friction, $\tau=-\left.\frac{\partial u}{\partial y}\right|_{y=0}$,

Nusselt number, $N u=-\left.\frac{1}{\theta(0, t)} \frac{\partial \theta}{\partial y}\right|_{y=0}=-\frac{1}{\theta(0, t)}$,

Sherwood number, $S h=\left.\frac{\partial C}{\partial y}\right|_{y=0}$.

It is worth to mention that the obtained solutions of concentration, temperature and velocity for cosine and sine are fully satisfied equation (6)-(8). The solutions obtained would be substituted to equations (6)-(8) and the validation occurs when the LHS of the equations is equal to the RHS. This verification shows the correctness of the obtained solutions. Then, in order to get a clear insight into the physical problem, the exact solutions are displayed with the help of graphical illustrations. Figure 2 shows concentration profile with different Sc. From the graph, increasing $S c$ will decrease the concentration. However, Figure 3 shows temperature profile with different $\mathrm{Pr}$. It shows that increasing $\mathrm{Pr}$ will decrease the temperature of fluid flow. This is in agreement with the physical fact that increasing $P r$, the viscosity of the fluid increases, the fluid become thicker which reduces heat transfer. Figures 4-8 show the effects of different parameters on the velocity profile. From Figure 4, it is observed that increasing $S c$ will decrease the velocity. It is found that $S c$ relates to the relative thickness of the mass transfer boundary layer, if $S c$ is increasing, the thickness of boundary layer would increase and will cause the velocity to decreases. While, Figure 5 shows the velocity profile with different $\mathrm{Pr}$. Increasing $\operatorname{Pr}$ will cause the velocity to decrease. Figure 6 shows velocity profile for different $\omega t$. The graph shows that increasing the $\omega t$ will cause the velocity to decrease. While, figures 7 and 8 show velocity profile with different $G r$ and $G m$. It is observed that velocity increasing with increasing the value of Gr. Physically this is possible because as $G r$ increases, the contribution from the buoyancy force near the plate becomes significant and 
hence a rise in velocity is observed. Similarly, when $G m$ is increases, the velocity is found increases.

$\mathrm{Nu}$ graph is plotted with different $\mathrm{Pr}$ in Figure 9. It shows that increasing $P r$ will increase $N u$. Figure 10 shows graph for $\tau$ for different $P r$. It is depicted from the graph that increasing $\operatorname{Pr}$ causes the $\tau$ decreases. However, Figure 11 shows graph of $S h$ against $t$ with different $S c$. The graph shows that increasing $S c$ will increase $S h$. It is worth to mention that, all presented graphs show that the solutions satisfy the boundary conditions of the problem.

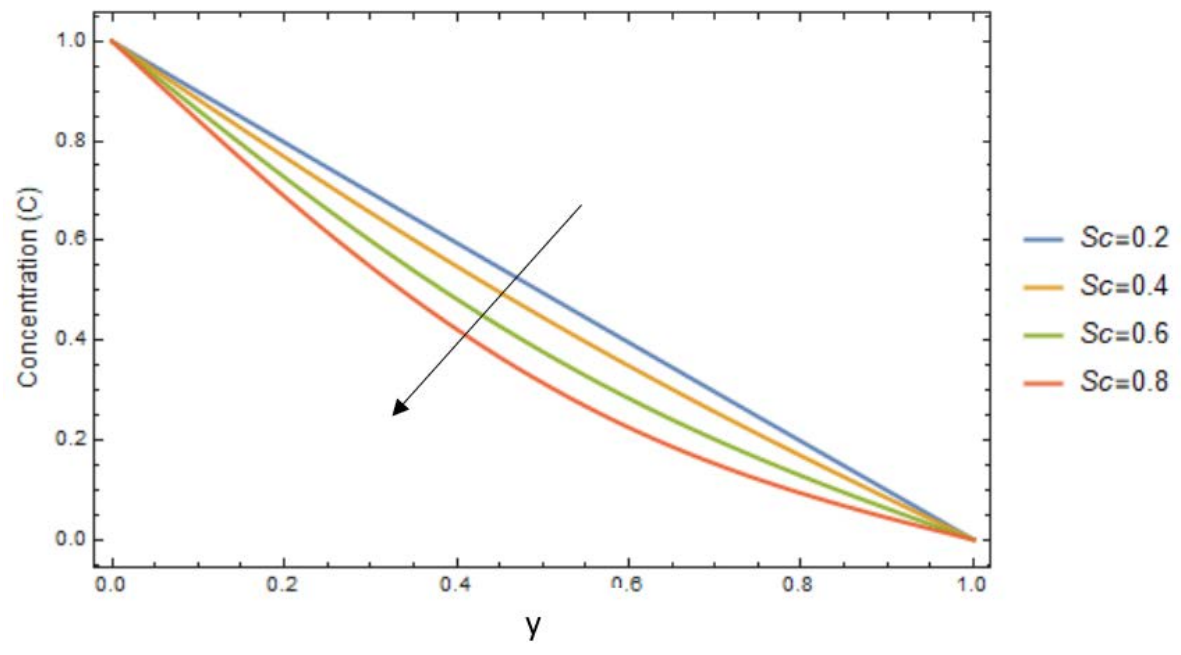

Figure 2. Concentration profile with different $S c$ when $t=0.1$

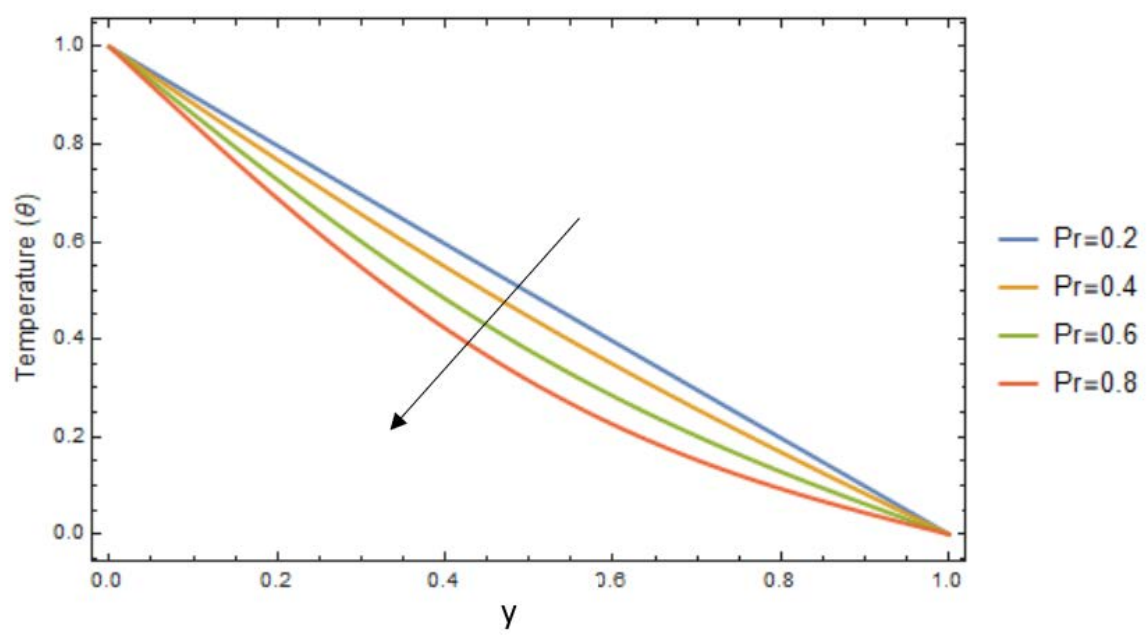

Figure 3. Temperature profile with different $\operatorname{Pr}$ when $t=0.1$ 


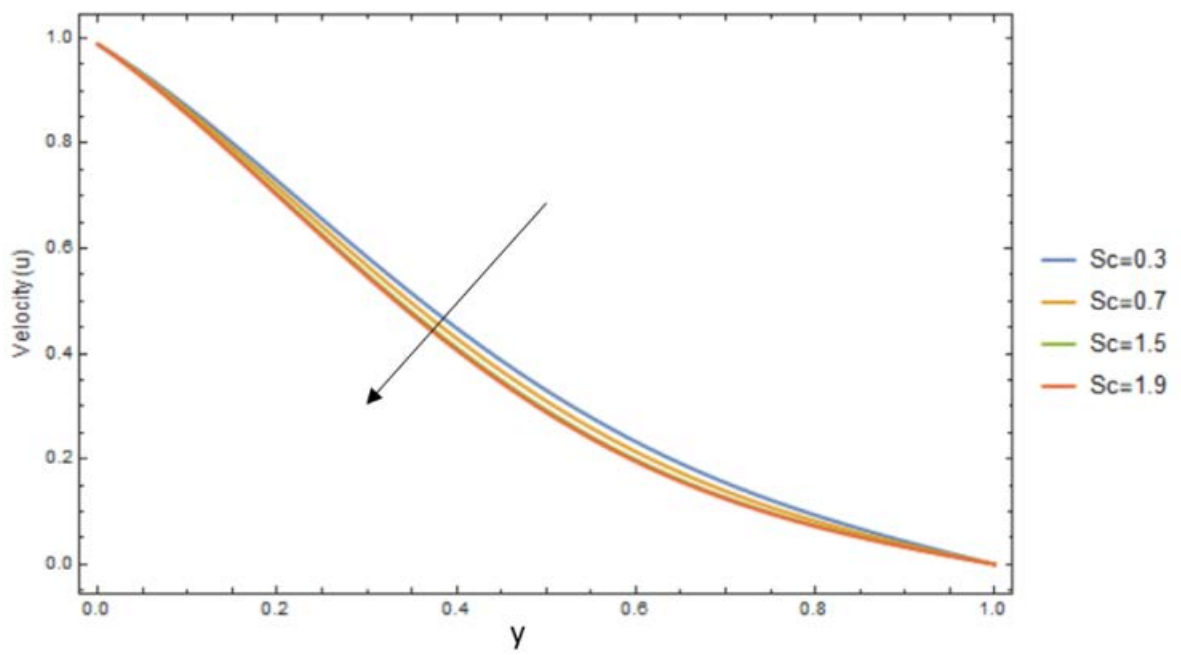

Figure 4. Velocity Profile with different $S c$ when $t=0.1$

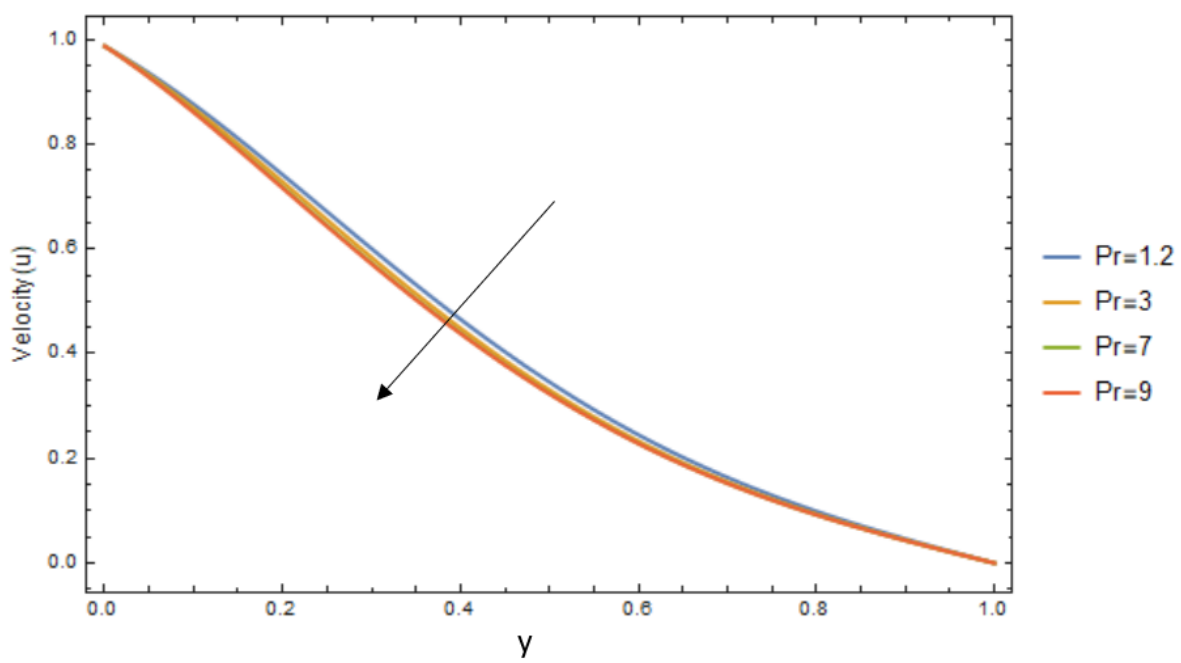

Figure 5. Velocity Profile with different $\operatorname{Pr}$ when $t=0.1$

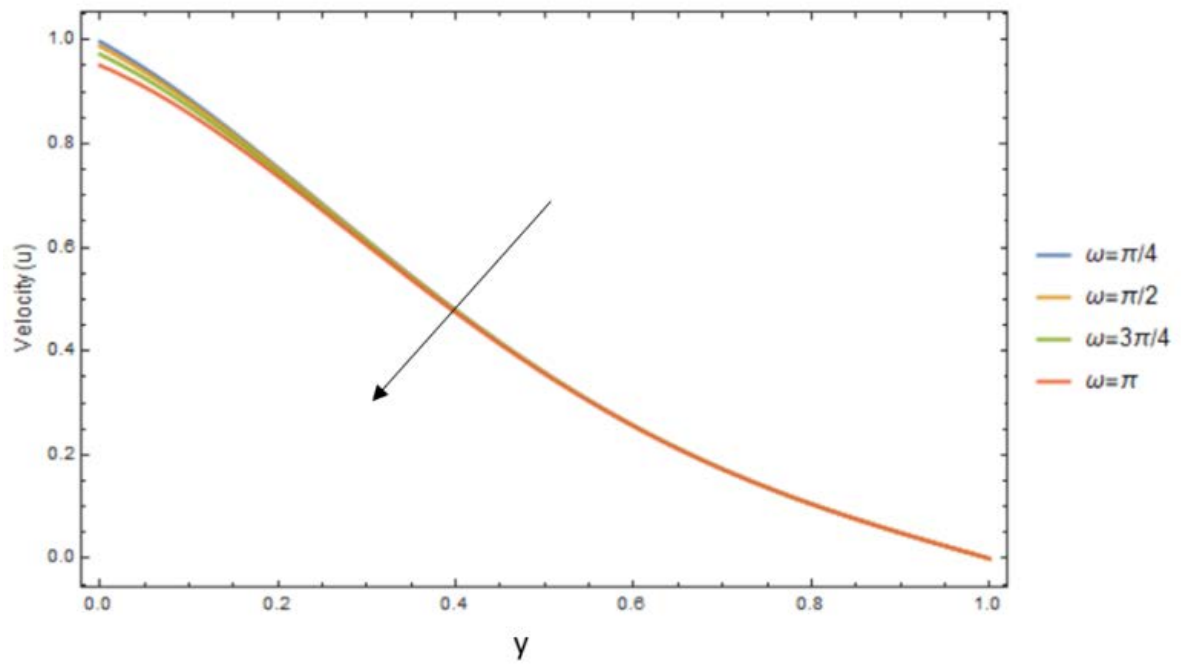

Figure 6. Velocity Profile with different $\omega$ when $\mathrm{t}=0.1$ 


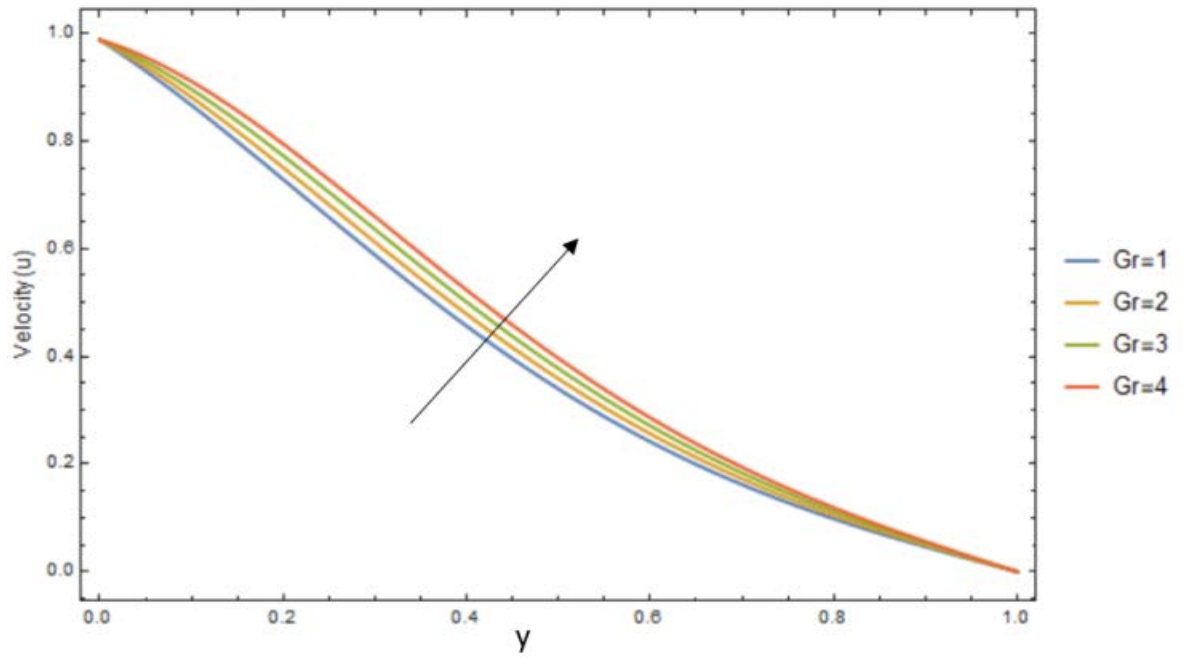

Figure 7. Velocity Profile with different $G r$ when $t=0.1$

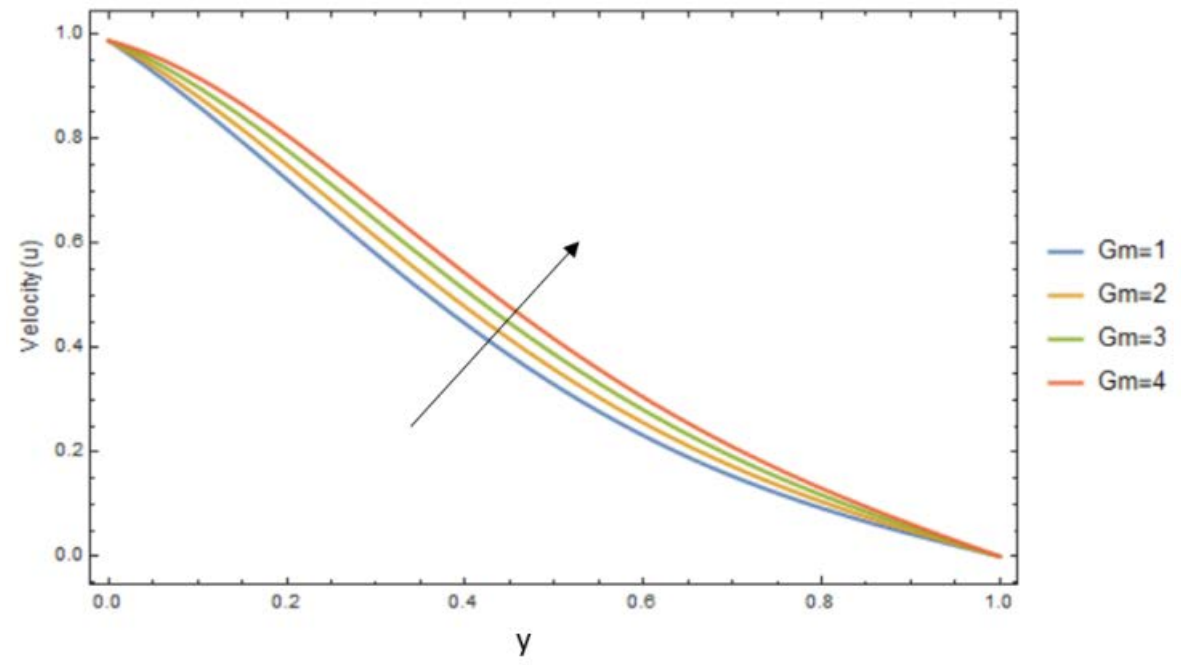

Figure 8. Velocity Profile with different $G m$ when $t=0.1$.

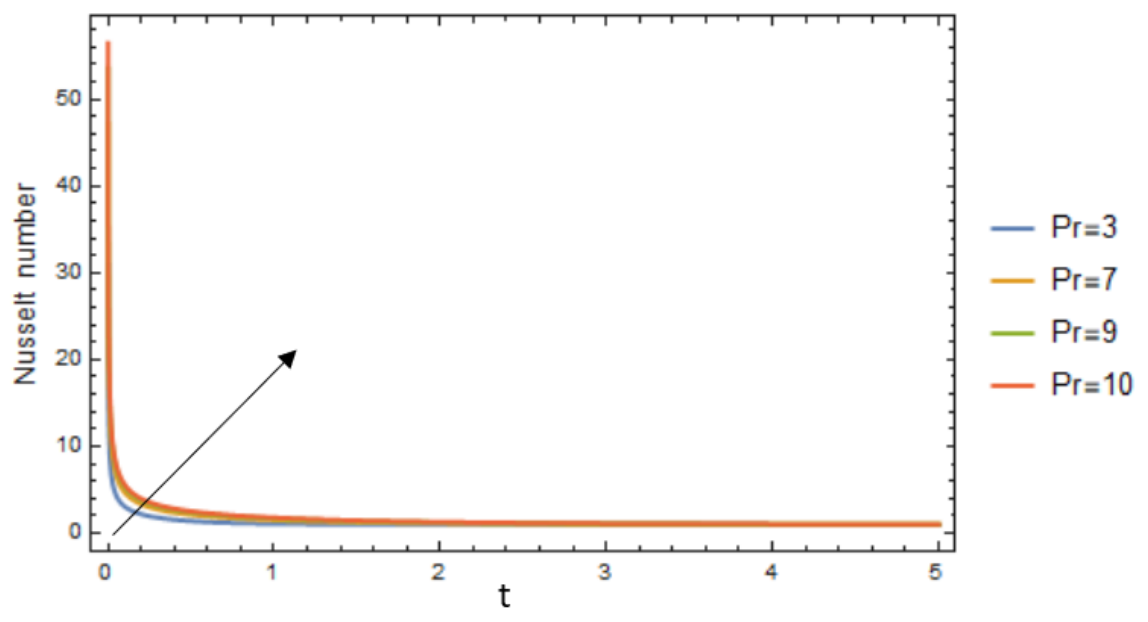

Figure 9. Nusselt number with different $P r$ 


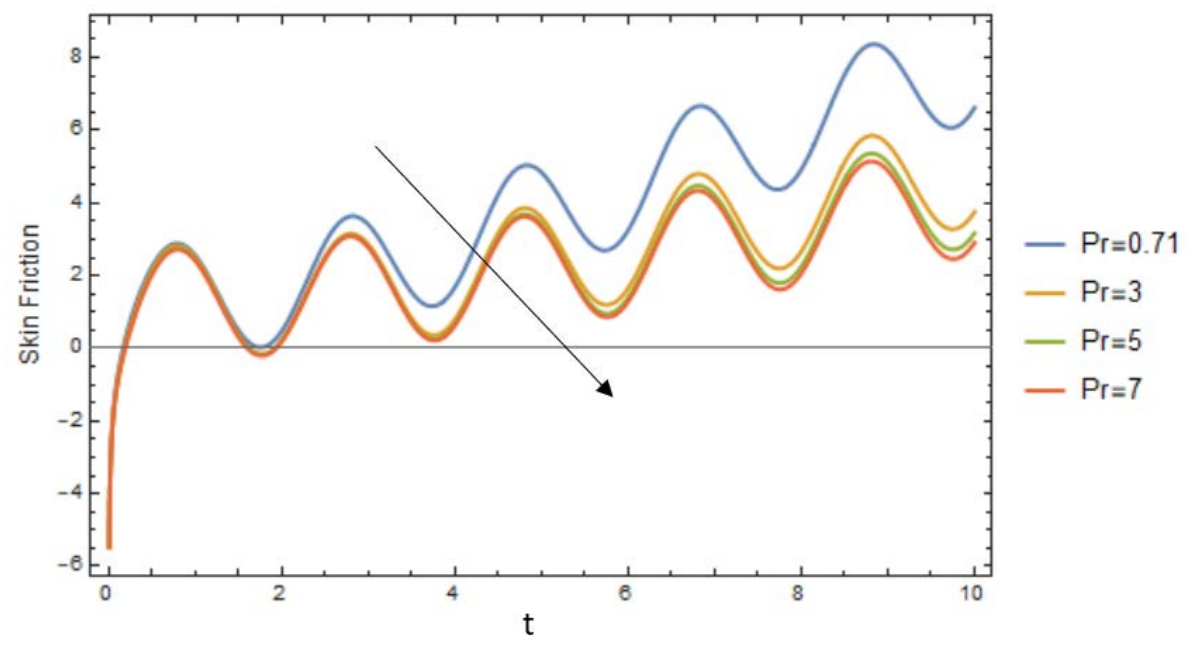

Figure 10. Skin Friction with different $P r$

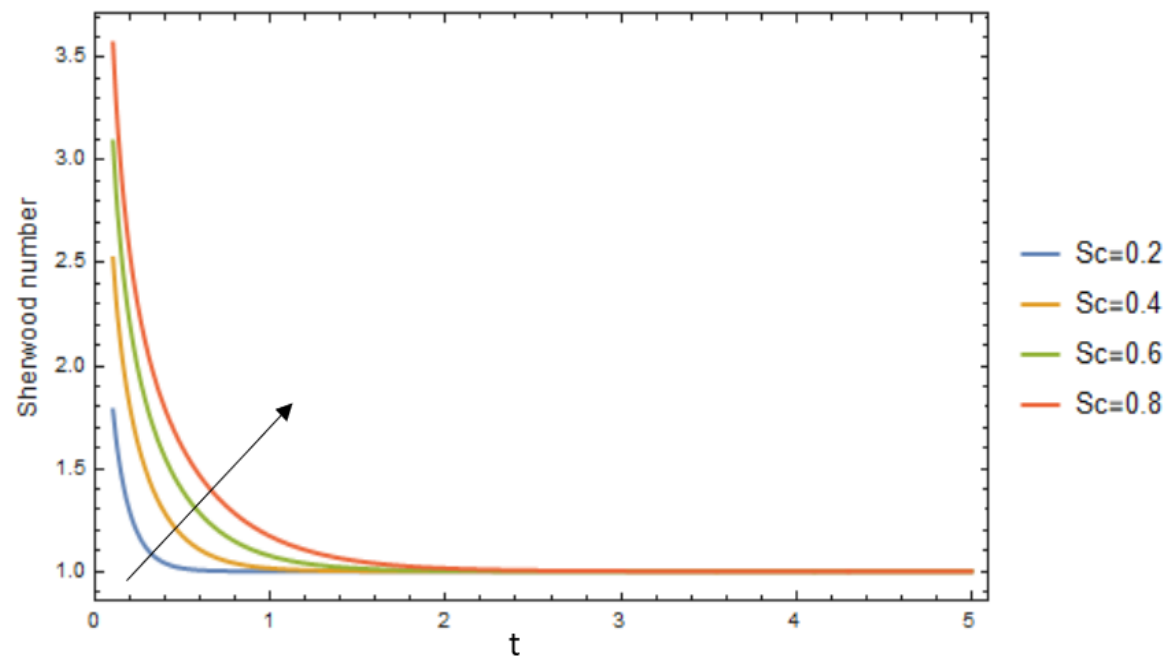

Figure 11. Sherwood number with different $S c$

\section{Conclusions}

An exact solution to the problem of oscillating free convection flow between two parallel plates with mass diffusion was investigated. The governing equation was reduced by using non-dimensional equations and solutions to the problem were obtained by using Laplace transform technique. The effects of various parameters on velocity, temperature and concentration profiles as well as skin friction, Nusselt and Sherwood number were graphically studied. From this study, it was found that increasing Schmidt number will decrease the concentration of the fluid but increased the Sherwood number. However, for Prandtl number, increasing Prandtl number decreased the temperature, velocity and skin friction but increased the Nusselt number. For oscillation parameter, it was found that increasing the oscillation parameter will decrease the velocity. However, increasing Grashof and mass Grashof number increased the velocity of the fluid flow.
Interestingly, the obtained solutions to velocity, temperature and concentration fully satisfied the non-dimensional equations (6)-(8).

\section{Acknowledgements}

The authors would like to acknowledge the Ministry of Education (MOE), UTM Zamalah and Research Management Centre-UTM, Universiti Teknologi Malaysia (UTM) for the financial support through vote numbers 5F004, 07G70, 07G72, $07 \mathrm{G} 76$ and 07G77 for this research.

\section{REFERENCES}

[1] Makinde, O. D., and P. Y. Mhone. "Heat transfer to MHD 
oscillatory flow in a channel filled with porous medium." Romanian Journal of physics 50, no. 9/10 (2005): 931.

[2] Paul, Trishna, B. K. Jha, and A. K. Singh. "Transient free convective flow in a vertical channel with constant temperature and constant heat flux on walls." Heat and mass transfer 32, no. 1-2 (1996): 61-63.

[3] Kundu, Balaram. "Semianalytical methods for heat and fluid flow between two parallel plates." Journal of Thermal Engineering 1, no. 3 (2015): 175-181.

[4] Hamza, M. M. "Free convection slip flow of an exothermic fluid in a convectively heated vertical channel." Ain Shams Engineering Journal (2016).

[5] Singh, A. K., and T. Paul. "Transient natural convection between two vertical walls heated/cooled asymmetrically." International Journal of Applied Mechanics and Engineering11, no. 1 (2006): 143-154.

[6] Narahari, M. "Effects of thermal radiation and free convection currents on the unsteady Couette flow between two vertical parallel plates with constant heat flux at one boundary." Wseas Transactions on heat and mass transfer 5, no. 1 (2010): 21-30.

[7] Jha, B. K., and A. O. Ajibade. "Free convection heat and mass transfer flow in a vertical channel with the Dufour effect." Proceedings of the Institution of Mechanical Engineers, Part E: Journal of Process Mechanical Engineering 224, no. 2 (2010): 91-101.

[8] Jha, Basant K., and Abiodun O. Ajibade. "Diffusion-thermo effects on free convective heat and mass transfer flow in a vertical channel with symmetric boundary conditions." Journal of Heat Transfer 133, no. 5 (2011): 052502.

[9] Bunonyo, K. W., E. Amos, and I. C. Eli. "Unsteady Oscillatory Couette Flow between Vertical Parallel Plates with Constant Radiative Heat Flux." (2018).

[10] Hossain, M. A., S. K. Das, and I. Pop. "Heat transfer response of MHD free convection flow along a vertical plate to surface temperature oscillations." International journal of non-linear mechanics 33, no. 3 (1998): 541-553.

[11] Chaudhary, R. C., and Arpita Jain. "Combined heat and mass transfer effects on MHD free convection flow past an oscillating plate embedded in porous medium." Romanian Journal of Physics 52, no. 5-7 (2007): 505-524.

[12] Khan, Ilyas, Kamran Fakhar, and Sharidan Shafie. "Magnetohydrodynamic free convection flow past an oscillating plate embedded in a porous medium." Journal of the Physical Society of Japan 80, no. 10 (2011): 104401.

[13] Reddy, T. Sudhakar, M. C. Raju, and S. V. K. Varma. "Unsteady MHD free convection oscillatory Couette flow through a porous medium with periodic wall temperature in presence of chemical reaction and thermal radiation." Int. J. Adv. Sci. Technol 1 (2011): 51-58.

[14] Falade, J. A., Joel C. Ukaegbu, A. C. Egere, and Samuel O. Adesanya. "MHD oscillatory flow through a porous channel saturated with porous medium." Alexandria engineering journal 56, no. 1 (2017): 147-152. 